\title{
TMS modulation of visual and auditory processing in the posterior parietal cortex
}

\author{
Nadia Bolognini $\cdot$ Carlo Miniussi · Silvia Savazzi • \\ Emanuela Bricolo $\cdot$ Angelo Maravita
}

Received: 23 September 2008 / Accepted: 17 April 2009 / Published online: 5 May 2009

(C) Springer-Verlag 2009

\begin{abstract}
Audio-visual stimuli typically yield faster responses than isolated modality-specific ones. This crossmodal speed advantage depends upon efficient multisensory integration mechanisms in the brain. Here, we used repetitive transcranial magnetic stimulation (rTMS) to address the role of the posterior parietal cortex, in particular of the inferior parietal lobule (IPL), in speeding up responses to crossmodal stimuli. The results show that rTMS over IPL impairs the response to contralateral modality-specific visual and auditory targets without affecting the response speed advantage following audio-visual targets. Furthermore, this speed advantage is subserved by a neural coactivation mechanism suggesting a summation in a given neural site. Control rTMS over V1 impaired only contralateral visual responses without affecting the response to auditory or audio-visual targets. These results suggest that
\end{abstract}

N. Bolognini $(\varangle) \cdot$ E. Bricolo $\cdot$ A. Maravita

Department of Psychology, University of Milano-Bicocca,

Via dell'Innovazione, 10, 20126 Milan, Italy

e-mail: nadia.bolognini@unimib.it

C. Miniussi

Department of Biomedical Sciences and Biotechnology,

National Institute of Neuroscience,

University of Brescia, Brescia, Italy

C. Miniussi

Cognitive Neuroscience Section,

IRCCS San Giovanni di Dio Fatebenefratelli, Brescia, Italy

S. Savazzi

Department of Neurological and Vision Sciences,

University of Verona, Verona, Italy

S. Savazzi

National Institute of Neuroscience,

University of Verona, Verona, Italy the response speed advantage for crossmodal targets is maintained in spite of the IPL interference that impairs modality-specific responses. The possible role of alternative sites for the audio-visual advantage, such as the superior colliculus, is discussed.

Keywords Multisensory $\cdot$ Superior colliculus · Parietal cortex $\cdot$ Auditory $\cdot$ Visual $\cdot$ rTMS

\section{Introduction}

In our daily life, a huge mass of sensory input animates our perceptual experience by impinging simultaneously on all our senses. The brain efficiently integrates such multisensory information in order to construct a congruent representation of the external space and organize appropriate motor acts (Stein and Meredith 1993; Stein 1998). One paradigmatic example of the advantage produced by multisensory integration is the redundant signals effect, i.e. the speeding up of responses to crossmodal as compared to modalityspecific spatial stimuli (Schröger and Widmann 1998; Maravita et al. 2008).

Several brain regions may account for a similar multisensory response speed advantage. At an early level, a critical role in audio-visual integration is played by the superior colliculus (SC) (Stein and Meredith 1993). Neurons in the deep layers of the cat's SC respond to stimuli from different sensory modalities, i.e. multisensory neurons (Meredith and Stein 1986), and their discharge is increased by multisensory stimuli, as compared to unisensory presentations, thus facilitating the animal's orienting towards a target (Stein et al. 1988). Critically, the response enhancement induced by multisensory stimuli in the SC is not merely due to the presentation of multiple instead of single targets, but is 
specific for crossmodal pairings, reflecting the peculiar multisensory integration properties of these neurons (Alvarado et al. 2007). In humans, as well as in animals, the SC may be critically involved in generating the crossmodal speed advantage of orienting responses (Maravita et al. 2005, 2008; Bolognini et al. 2007).

However, substantial audio-visual processing is also occurring in the cortex. In particular, the posterior parietal cortex (PPC) is a heteromodal region of sensory convergence that contains neurons responding to isolated visual and auditory stimuli (Bushara et al. 1999; Bremmer et al. 2001b) and multisensory neurons (Duhamel et al. 1991; Andersen 1997; Graziano and Gross 1998). These latter cells may be the ideal locus for multisensory integration, thus contributing to supramodal cognitive functions such as spatial attention and spatial awareness (Bushara et al. 1999; Bremmer et al. 2001a; Macaluso and Driver 2005; Nachev and Husain 2006). These features render the PPC a further cortical site candidate for the crossmodal speeding up typical of the audio-visual stimuli (Gondan et al. 2005).

Here, we investigated whether interfering with the PPC by means of low-frequency repetitive transcranial magnetic stimulation (rTMS) (Chen et al. 1997) causes a disruption of the typical response speed advantage observed with crossmodal targets or if any crossmodal facilitatory effect is resistant to the interference with this brain region. rTMS was applied over the PPC, using stereotaxic coordinates corresponding to the inferior parietal lobule (IPL, Brodmann's area, BA 40), typically associated with multimodal spatial representation (Macaluso and Driver 2005). The causal role of this parietal region in multisensory and unisensory processing was then assessed with a reaction time task that typically produces faster responses to crossmodal as compared to unisensory targets (Miller 1982).

As a control condition, we stimulated the right occipital cortex (V1). The prediction is that if the IPL has a necessary role in facilitating the detection of multisensory audiovisual stimuli, rTMS interference over this area (but not over unimodal V1) should abolish the typical facilitation of reaction times that follows audio-visual presentations.

\section{Experimental procedure}

\section{Participants}

Thirteen right-handed healthy participants (age 23-41 years) took part in the study. They were naive to the experimental procedure and to the purpose of the experiment. None of the participants had neurological, psychiatric, or other relevant medical problems or had any contraindication to rTMS (Wassermann 1998; Machii et al. 2006). All participants gave written informed consent and received course credit for their participation. The protocol was carried out in accordance with the ethical standards of the Declaration of Helsinki (BMJ 1991; 302:1194) and it was approved by the ethical committee of the University of Milano-Bicocca.

Methods

Participants sat in a dimly lit, silent room, at a distance of $47 \mathrm{~cm}$ in front of a PC monitor (Samsung SyncMaster $1200 \mathrm{NF}$ ). The screen background was dark grey with a luminance of $0.01 \mathrm{~cd} / \mathrm{m}^{2}$. Each trial started with the presentation of a central fixation circle. After $400 \mathrm{~ms}$ a target (visual, auditory or audio-visual) or a catch trial was randomly presented at the centre of a guide box made up of nine flickering grey squares. Target luminance was set at $1.9 \mathrm{~cd} / \mathrm{m}^{2}$. The visual target $(1.2 \times 1.2 \mathrm{deg}$ of visual angle) was a red square presented at $20^{\circ}$ from fixation. The auditory targets consisted of a burst of white noise, delivered from external loudspeakers placed at the same height as the visual target, on the left or right side of the monitor (see Fig. 1). During catch trials there was no sound and no visual target.

The participants were asked to respond as soon as possible at the onset of the target by pressing the space bar of the $\mathrm{PC}$ with their right index finger and to refrain from responding to catch trials. The following stimulus conditions were randomly presented: Single auditory (left or right); single visual (left or right); crossmodal audio-visual (left or right). Audio-visual stimuli were presented in the left or right hemifield, but they were always spatially congruent (i.e. an auditory stimulus presented simultaneously to the visual target at the same side) since a preliminary work from our laboratory has shown that spatial coincidence yields maximum audiovisual integration in this paradigm (Maravita et al. 2008). There were 48 trials for each type of stimulus ( 24 trials for each side of stimulation), in addition to 48 catch trials. The total number of trials (288) was equally distributed in four experimental blocks, presented in random order.

The task was repeated for each subject in three different sessions (A, B, C), counterbalanced across participants and conducted over different days (the inter-sessions interval was at least 1 day): a baseline session with no rTMS; and two rTMS sessions (Fig. 1). In both rTMS sessions, the 1$\mathrm{Hz}$ stimulation was applied for $20 \mathrm{~min}$ before the subject was tested on the task. The duration of the task was of about $12 \mathrm{~min}$ therefore each experimental session lasted about $32 \mathrm{~min}$.

\section{rTMS protocol}

Low-frequency $(1 \mathrm{~Hz})$ off-line rTMS was delivered using a Magstim Super Rapid magnetic stimulator (Magstim, Whitland, UK) and a figure-of-eight coil (70 $\mathrm{mm}$ diameter). It 
A

Day A - No Stimulation

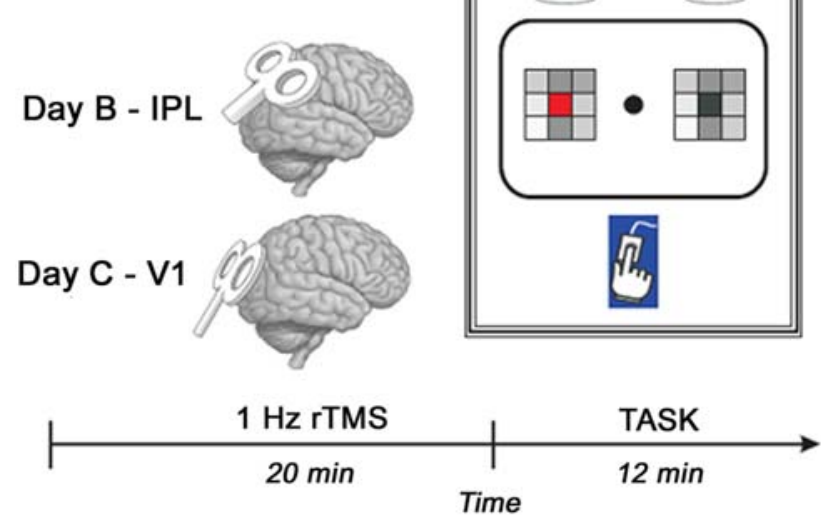

B
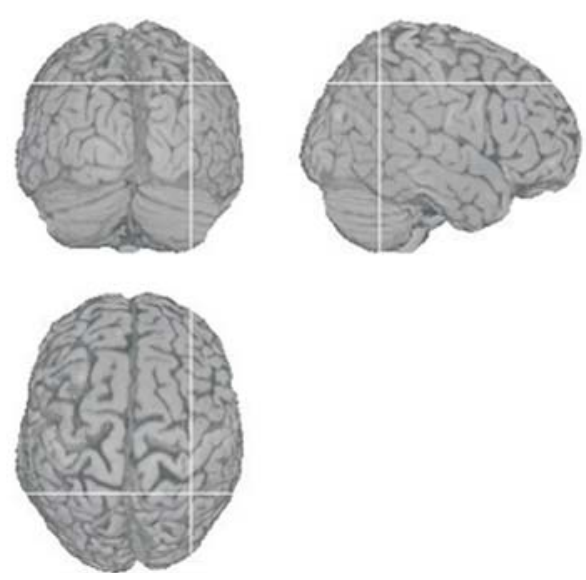

Fig. 1 a On the left side the experimental conditions are illustrated (no-stimulation, IPL- and V1-rTMS). In the conditions where rTMS was applied, the figure-of-eight coil was placed in the position depicted in each figure. On the right side the stimulus display is illustrated. The visual stimulus appeared at the centre of an array of nine flickering squares, on the right or the left of fixation and could or could not be accompanied by a spatially coincident auditory stimulus (see depicted loudspeaker). b Posterior, top and lateral views of the stimulated site depicted on a standard template from MRIcro (v1.39; http://www.mricro.com). The cross-hairs indicate the estimated site stimulated by rTMS. The Talairach coordinates of this site, which were calculated by the SofTaxic Navigator system, are $X=40, Y=-52$ and $Z=44$; corresponding to a right PPC site overlying the IPL (BA 40)

has been shown that using off-line rTMS it is possible to transiently modulate neural excitability with the net effect dependent on the stimulation frequency. Generally, from a physiological point of view, low frequency $(\leq 1 \mathrm{~Hz})$ results in inhibition in the stimulated area (Chen et al. 1997). Similar effects have also been found in behavioural experiments (Pascual-Leone et al. 2000; Lewald et al. 2002; Romero et al. 2002; Knecht et al. 2003; Merabet et al. 2004; Bolognini et al. 2007; Cappelletti et al. 2007).

TMS stimuli were delivered at a fixed intensity, $65 \%$ of the maximum output of the stimulator. These stimulus parameters were compatible with the aim of the present experiment, which was to interfere with the normal functioning of stimulated areas (Bolognini and Maravita 2007).

The targeted stimulation sites were either the right IPL (see Fig. 1b) in the experimental condition, or the right primary visual cortex (V1) in the control condition. V1 was chosen in order to compare the effects of the stimulation of a unisensory (visual) versus multisensory (IPL) brain area over the response to modality-specific or crossmodal stimuli.

We localized the targeted areas using the SofTaxic Evolution navigator system (Version 1.0, http://www. emsmedical.net). This system allows the reconstruction of cerebral cortex in Talairach coordinates, with an accuracy of $\sim 1 \mathrm{~cm}$, on the basis of digitized skull landmarks (nasion, inion and two preauricular points) and other 50 uniformly distributed points that are mapped on the scalp (3D Fastrak Polhemus digitizer). An estimation of the single subject's cerebral volume is obtained by "Point-based Warping" to an MRI template and a 3D virtual reconstruction based on the points recorded from the subject's scalp.

Following this procedure we localized for each subject the right IPL (Fig. 1b), on average, on Talairach coordinates $X=40, Y=-52, Z=44$, (BA 40) (Talairach and Tournoux 1988). The choice of this stimulation site was based upon previous fMRI work (Bushara et al. 1999). Furthermore, the right $\mathrm{V} 1$ corresponded on average to coordinates $X=19, Y=-97, Z=1$ (BA 17) (Talairach and Tournoux 1988). The coil, was positioned on the correct site on each experimental block, and was supported and held in place by a mechanical device.

\section{Results}

The mean reaction time (RTs) to unimodal and crossmodal stimuli was measured separately for each hemifield and then analysed with a repeated measure analysis of variance (ANOVA) with session (baseline, post-IPL-rTMS, post V1-rTMS), hemifield (right vs. left hemifield) and stimulus (auditory, visual, audio-visual) as main factors. Whenever necessary, pair-wise comparisons were conducted using the Newman-Keuls test. Mean percentages of omissions and false allarms in each experimental session are reported in Table 1.

First, we found a significant main effect of the factor hemifield $[F(1,12)=14.59, P<0.002]$, due to slower RTs to left-sided-stimuli (contralateral to the stimulated hemisphere in the rTMS sessions) $(372 \mathrm{~ms})$ than right ones (361 ms, $P<0.002)$. The significant factor stimulus $[F(2,24)=19.86, P<0.0001]$ was due to a general speedup of RTs following audio-visual targets $(323 \mathrm{~ms})$ as compared to modality-specific ones (auditory $=380 \mathrm{~ms}$ and 
Table 1 Mean percentages of omissions and false allarms in each experimental session

\begin{tabular}{|c|c|c|c|c|c|c|}
\hline & \multicolumn{3}{|c|}{ Left hemifield } & \multicolumn{3}{|c|}{ Right hemifield } \\
\hline & Auditory (\%) & Visual (\%) & $\begin{array}{c}\text { Audio-visual } \\
(\%)\end{array}$ & Auditory (\%) & Visual (\%) & $\begin{array}{c}\text { Audio-visual } \\
(\%)\end{array}$ \\
\hline \multicolumn{7}{|c|}{ Baseline } \\
\hline Omissions & 2 & 0 & 0 & 2 & 0 & 1 \\
\hline False allarms & \multicolumn{3}{|c|}{4} & \multicolumn{3}{|c|}{5} \\
\hline \multicolumn{7}{|c|}{ Post-IPL-rTMS } \\
\hline Omissions & 1 & 0 & 0 & 1 & 1 & 0 \\
\hline False allarms & \multicolumn{3}{|c|}{7} & \multicolumn{3}{|c|}{3} \\
\hline \multicolumn{7}{|c|}{ Post-V1-rTMS } \\
\hline Omissions & 3 & 1 & 0 & 2 & 2 & 0 \\
\hline False allarms & \multicolumn{3}{|c|}{5} & \multicolumn{3}{|c|}{3} \\
\hline
\end{tabular}

A

Fig. 2 a Mean RTs to each stimulation condition (unimodal auditory and visual, bimodal audio-visual) in the left and right sides of space, in the three experimental sessions. Error lines illustrate the standard error. b Difference in the mean RT $(\Delta \mathrm{RT})$ between the right and left sides of space for each stimulus condition in the three experimental sessions

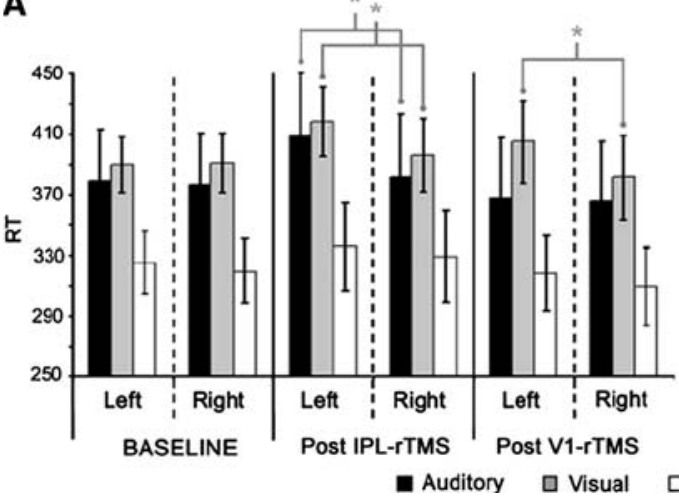

B

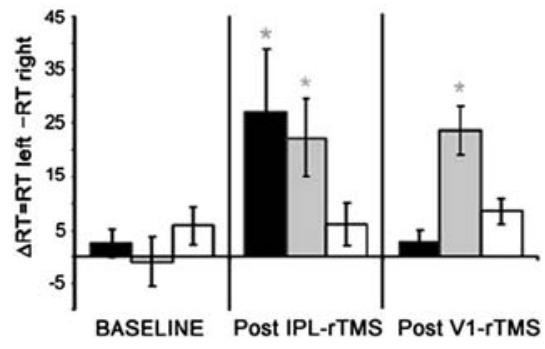

visual $=397 \mathrm{~ms}, \quad P<0.0002)$, highlighting a significant crossmodal speed advantage in all sessions.

The session by hemifield interaction $[F(2,24)=5.62$, $P<0.009$ ] showed that right IPL and V1 rTMS yielded overall longer RTs in the left hemifield as compared to the baseline, where no hemifield effect was present (baseline: right $=362 \mathrm{~ms}$ vs. left $=365 \mathrm{~ms}, P=0.7$; post-IPL-rTMS: right $=369 \mathrm{~ms}$ vs. left $=388 \mathrm{~ms}, \quad P<0.0001$; post-V1rTMS: right $=352 \mathrm{~ms}$ vs. left $=364 \mathrm{~ms}, P<0.006$ ).

The critical result was the significant interaction session $\times$ hemifield $\times$ stimulus $[F(4,48)=4.13, P<0.006]$. This was due to the different effect of rTMS in the processing of contralateral stimuli as compared to the ipsilateral ones (see Fig. 2a and the interhemispheric difference illustrated in Fig. 2b), which was present only for modality-specific stimuli. In particular, no difference between left and right hemifields was present in the baseline session (auditory: left $=379$ vs. right $=376$; visual: left $=391$ vs. right $=390$; audio-visual: left $=325$ vs. right $=319$; all $P>0.3$ ). By contrast, IPL-rTMS increased the response to both modality-specific auditory (left $=409$ vs. right $=382 \quad P<0.0001$ ) and visual (left $=418$ vs. right $=396 \quad P<0.0001)$ stimuli without affecting the response speed advantage to audio-visual targets (left $=336$ vs. right $=329 P>0.3$ ). Instead, V1-rTMS increased the response to unisensory visual stimuli only (left $=405$ vs. right $=381 P<0.003$ ) but did not significantly affect the response speed to unisensory auditory stimuli (left $=368$ vs. right $=365 ; P>0.6)$ and the RT advantage to audiovisual targets $(\mathrm{left}=318$ vs. right $=310 ; P>0.09)$ in both hemifields. Noteworthy, further direct comparisons confirmed that the slowing of contralateral modality-specific responses induced by rTMS was significantly different even when compared with the baseline for both modalities following IPL-rTMS (auditory: baseline $=379 \mathrm{~ms}$ vs. postIPL-rTMS $=409 \mathrm{~ms}, P<0.0001$; visual: baseline $=390 \mathrm{~ms}$ vs. post-IPL-rTMS $=418 \mathrm{~ms}, \quad P<0.0001)$ and for the visual modality only following V1-rTMS (visual: baseline $=405 \mathrm{~ms}$ vs. post-V1 $\mathrm{rTMS}=390 \mathrm{~ms}, P<0.02$ ) (see Fig. 2; Table 2). 
Table 2 Summary of all effects from ANOVA of RTs: $F$ and $P$ values

\begin{tabular}{lll}
\hline Main effects & $F$ & $P$ level \\
\hline Session & $(2,24)=0.73$ & 0.5 \\
Hemifield & $(1,12)=14.59$ & 0.002 \\
Stimulus & $(2,24)=19.86$ & 0.0001 \\
Session $\times$ hemifield & $(2,24)=5.62$ & 0.009 \\
Session $\times$ stimulus & $(4,48)=0.55$ & 0.7 \\
Hemifield $\times$ stimulus & $(2,24)=2.57$ & 0.1 \\
Session $\times$ hemifield $\times$ Stimulus & $(4,48)=4.13$ & 0.006 \\
\hline
\end{tabular}

Effects of cortical interference: neural co-activation versus probability summation?

In all experimental sessions we observed that left and right audio-visual stimuli yielded significantly faster responses than isolated unisensory ones. The critical finding was that, even after interference on both unisensory stimuli by parietal rTMS, the crossmodal speed advantage remained unaltered and an increased multisensory gain, i.e. difference in the RT between the average response to the unisensory events and the crossmodal event, was observed.

The nature of the response facilitation induced by multiple stimuli can be inferred with a mathematical procedure introduced by Miller (1982). This procedure determines whether the speed advantage with redundant stimuli (i.e. the Redundant Signal Effect, RSE) is likely to follow a probabilistic facilitation resulting from signals travelling through separate information channels, i.e. race model (Raab 1962), or to co-activation in a neuronal pool where redundant signals converge (co-activation model). The latter mechanism implies a specific neural structure, whereas the former simply postulates that the fastest among the signals conveyed by different channels triggers the response.

In order to discriminate between these two explanations for the RSE, Miller (1982) has proposed the "race inequality test". This procedure compares the cumulative distribution frequencies (CDFs) of RTs to signals travelling through different sensory channels, depending on whether single or double stimuli are presented.

The inequality sets an upper limit for the cumulative probability of a response at any time $(t)$, given redundant targets: $\quad P\left(\mathrm{RT} \leq t \mid T^{\mathrm{V}}\right.$ and $\left.T^{\mathrm{A}}\right) \leq P\left(\mathrm{RT} \leq t \mid T^{\mathrm{V}}\right)+$ $P\left(\mathrm{RT} \leq t \mid T^{\mathrm{A}}\right)$, where $P\left(\mathrm{RT} \leq t \mid T^{\mathrm{V}}\right.$ and $\left.T^{\mathrm{A}}\right)$ is the cumulative probability of a response, given a redundant target (such as an audio-visual target, as in the present experiment) $\left(T^{\mathrm{V}}\right.$ and $\left.T^{\mathrm{A}}\right) ; P\left(\mathrm{RT} \leq t \mid T^{\mathrm{V}}\right)$ is the cumulative probability of a response, given a visual target $\left(T^{\mathrm{V}}\right)$ and $P\left(\mathrm{RT} \leq t \mid T^{\mathrm{A}}\right)$ is the cumulative probability of a response, given an auditory target $\left(T^{\mathrm{A}}\right)$. If the upper boundary of the inequality is violated at any percentile, a race model cannot explain the data and a co-activation model is more likely.

Here, we followed this procedure in order to assess, in every experimental session, whether any response speed advantage was likely due to the integration of signals within a given neural site. Therefore, in each experimental session, separately for each hemifield, the summed CDFs of the RTs to the two modality-specific stimuli were subtracted from the CDFs of the RTs to audio-visual stimuli for each participant. A positive difference indicates that the upper boundary set by Miller's inequality is violated, and that a race-model probabilistic mechanism can be ruled out. The difference between the CDFs of the RTs to crossmodal stimuli and the summed CDFs of the RTs to modality-specific stimuli was tested with one-sample $t$ tests at corresponding percentiles across all participants (Miller 1982). As stated by Miller, this method enables to determine which portion of the curve indicating a violation of the inequality is statistically different from zero, and a significant difference at any point of the curve is enough to rule out any independent-channels explanation for the RSE (Miller 1982).

Our results showed that, in every session, the RSE violated the Miller's inequality in both hemifields, thus suggesting that under these conditions the audio-visual benefit in reaction times was likely subserved by a neural co-activation mechanism, rather than by an independent-channels mechanism such as the race model (Fig. 3). Importantly, this was true even after IPL-rTMS where the size of violation was larger than that of any other session, perhaps as consequence of strongest slowing of modality-specific responses.

\section{Discussion}

This study investigated the critical involvement of the human inferior parietal lobule (IPL) in the spatial processing of modality-specific and crossmodal stimuli. Overall, results indicate that interfering with the activity of the right IPL by rTMS causes selective spatially lateralized modality-specific impairments across different sensory modalities, namely visual and auditory, without affecting their multisensory integration. Furthermore, such a residual advantage for multisensory stimuli is always explained by a neural co-activation mechanism (Miller 1982), meaning that it is likely to occur in a given neural structure of multisensory convergence.

Recent brain imaging studies in humans have revealed that multisensory spatial processing is subserved by a complex cortical-subcortical circuit. In particular, multisensory areas within the PPC and the SC are increasingly being implicated in tasks involving the localization of crossmodal 


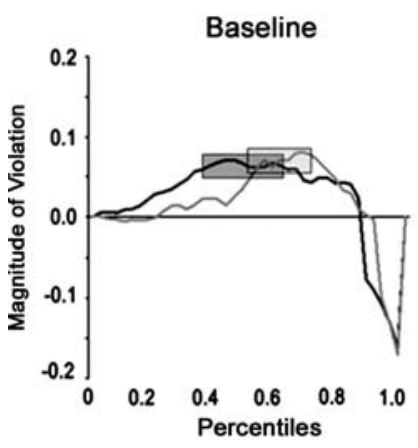

Fig. 3 Magnitude of the Miller inequality violation in the baseline and post IPL-rTMS and post-V1-rTMS sessions as assessed separately in each hemifield. In the $y$ axis the difference between the cumulative probability of RT on crossmodal target trials, and the summed cumulative probability of response on modality-specific targets, at different
Post IPL-rTMS

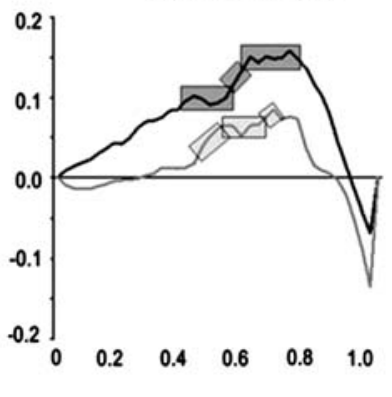

Right Hemifield
Post V1-rTMS

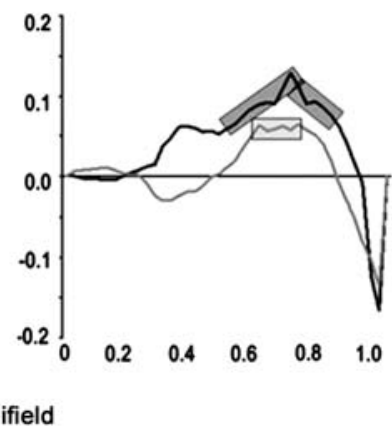

percentile values ( $x$ axis), is reported. Values above the zero line indicate a violation of the inequality. The shaded areas over the individual curves indicate the percentiles at which a significant violation was found by one-sample $t$ test

stimuli (Calvert et al. 2001; Macaluso and Driver 2005; Stein and Stanford 2008). Both the parietal cortex and the $\mathrm{SC}$ receive projections from different sensory systems and contain multisensory neurons (Hyvärinen1981; Graziano and Gross 1998; Schlack et al. 2005; Stein and Stanford 2008), thus suggesting their potential importance for the synthesis of different sensory inputs. Moreover, neurophysiologic and behavioural studies in cats have shown that overt multisensory orientating behaviour crucially depends on a cross-talk between the association (i.e. anterior ectosylvian) cortex and the SC (Jiang et al. 2001, 2002). It has been proposed that multisensory integration at the cortical level may play a different role from that in the SC and may be governed by slightly different rules (Wallace et al. 1992; Stein 1998).

More in details, our first finding is that rTMS interference over IPL impairs responses to isolated visual or auditory stimuli, but not to combined audio-visual stimuli; these modality-specific impairments are restricted to the hemifield contralateral to the rTMS side.

This result is in line with neuroimaging evidence showing modality-specific, besides multimodal, activations within different brain areas, including multiple subregions of the PPC (Andersen 1997; Bushara et al. 1999; Downar et al. 2000; Bremmer et al. 2001b). It appears that some modality-specific (i.e. unimodal) areas may exist quite close to regions representing multisensory space within the PPC (Bushara et al. 1999; Downar et al. 2000; Bremmer et al. 2001b). Therefore, the modality-specific auditory and visual spatial deficits observed here after rTMS over IPL could be due to the selective disruption of neighbouring unimodal spatial representations for each of the two modalities within the parietal cortex, without disruption of multisensory integrative effects in IPL.

There are different multisensory cortical areas that might provide a potential substrate for the residual audio-visual

effects on reaction times. These areas include the frontal premotor cortex, which has been implicated in multisensory spatial attention to peripheral locations (Calvert et al. 2001; Macaluso and Driver 2005), and the superior temporal sulcus (Calvert et al. 2001) that process both simple (such as moving visual gratings) and meaningful stimuli (Beauchamp 2005).

Another explanation for the persistence of multisensory effects is that other structures beyond the cortex may have been able to compensate for any local interference with the parietal activity. In particular, the SC might be critically implicated in speeding responses to bimodal stimuli after cortical disruption. There are different reasons supporting this proposal. First, the SC is known to be vital for both reflexive attention and multisensory integration (Stein 1998). Moreover, it is known that the SC is a likely locus of neural co-activation in the response to redundant targets. Indeed, if visual stimuli that selectively activate short-wave sensitive retinal cones (S cones), to which the SC is blind, are used, any multisensory effect is typically explained by a "race model", not by a neural co-activation mechanism (Maravita et al. 2008). Therefore, since the crossmodal speed advantage exceeded the so-called race model in every experimental session, including after IPL-rTMS (as well as V1-TMS), this would suggest that the observed audio-visual integration occurs in a neural site that is different than the stimulated IPL region, and we suggest that it could possibly be the SC.

Furthermore, data from the cat suggest that when crossmodal spatially coincident stimuli are presented, the animal orienting responses are significantly faster, following an increased discharge of a number of multisensory single cells within the SC (Stein and Meredith 1993; Stein 1998). Noteworthy, this multisensory enhancement is particularly strong when weakly effective stimuli are combined, whereas it is much reduced or even absent for the combination of 
more salient stimuli, a functional feature known as inverse effectiveness rule (Stanford and Stein 2007). Although a strict parallel between physiological responses of single neurons and behavioural effects in humans should be taken cautiously, it is suggestive to think that by disrupting the response to modality-specific stimuli following IPL interference may have increased the advantage for crossmodal combinations, compatibly with the inverse effectiveness rule observed in SC single neurons.

On a broader behavioural perspective, it has been proposed that the magnitude of multisensory integration is a measure of the relative physiological salience of an event. Given that sensory stimuli compete for attention and for access to the motor system that generates reactions to them, then the potential consequence of multisensory enhancement is an increased likelihood of detecting and/or initiating a response to the source of the signal. As a consequence, the extent to which multisensory integration aids the detection of an event has a direct positive effect on the speed with which a response can be generated (Stein and Stanford 2008). Our data seem to support this view: When the subjective salience of unisensory events is weakened by parietal disruption, this was followed by a persistent benefit of combining crossmodal information. ${ }^{1}$ It is for additional experiments to further explore this observation by using adequate techniques. For instance, using the diffusion superposition model (Schwarz 1994) would allow to predict effects, such as the inverse effectiveness, assessing the existence of greater co-activation effects for combining weaker signals. However, the adjustment of the model parameters requires a systematic variation of the temporal interval between unimodal stimuli, at odds with the present work where bimodal stimuli were always simultaneously presented.

Noteworthy, the observed modality-specific spatially lateralized impairments after IPL interference are also evocative of neglect-like symptoms. Patients with damage to the right IPL frequently show a reduced response to stimuli located in the space contralateral to the damaged hemisphere (Vallar et al. 2003). Crucially, these behavioural deficits can occur across different sensory modalities. For instance, patients with visual neglect can often manifest additional contralesional auditory deficits, which correlate in their severity with the visual impairments (Pavani et al. 2003; Clarke and Thiran 2004; Brozzoli et al. 2006). Moreover, the persistence of crossmodal facilitation in spite of modality-specific impairments is also in line with neuro-

\footnotetext{
${ }^{1}$ In fact, there was even a tendency towards a greater violation of the race inequality after degrading the response to unimodal with parietal rTMS. This finding could suggest a stronger multisensory effect, although, as such, it has not been demonstrated that a greater violation of the inequality implies a change of any precise neural response.
}

psychological evidence showing that multisensory integrative processes are overall preserved after parietal and/or occipital lesions and they can even overcome the unisensory deficits caused by the cortical damage, most likely through early sensory processing within subcortical brain sites such as the SC (Frassinetti et al. 2005).

Finally, we found that stimulation of V1 specifically impaired responses to contralateral visual targets. This effect is in line with previous studies revealing visual suppression by occipital TMS (Amassian et al. 1989; Romei et al. 2007): the slowing down of visual responses after V1 rTMS might represent a perceptual effect due to the interference with early modality-specific sensory processing in V1. On the other hand, given the extensive literature demonstrating that there is substantial attentional modulation even in early visual cortex (Duncan et al. 1997; Kastner and Ungerleider 2000, 2001), V1 may also be crucial for some aspects of reflexive visual orienting.

The fact that V1-rTMS did not disrupt audio-visual integration, again supports the possibility that substantial multisensory integration may occur even when the integrity of primary cortices is compromised (Stein 2005).

In conclusion, our study highlighted the importance of the IPL in the analysis of modality-specific visual and auditory stimuli. Furthermore, it showed that the impairment of modality-specific processing may occur in spite of preserved crossmodal facilitation. This evidence suggests that, at least for orienting to simple unstructured stimuli, the advantage for multisensory stimuli may overcome a significant impairment in the processing of modality-specific stimuli. This may be due to the occurrence of substantial multisensory processing in structures other than the IPL, such as the SC. The existence of effective mechanisms of spatial orienting persisting after the interference with parietal and occipital areas may have a clinical relevance and could be an unique opportunity for implementing effective rehabilitation procedures after brain damage (Bolognini et al. 2005). Crossmodal stimuli could effectively improve the impaired response to lateralized modality-specific stimuli observed in brain-damaged patients suffering from neglect or hemianopia, thanks to spared processing in spared multisensory structures.

Acknowledgments This research was supported by grants from the Ministero Italiano dell'Università e della Ricerca Scientifica (MIUR) to AM. We thank A. Pascual-Leone and B.E. Stein for insightful discussions and Carlo Toneatto for technical assistance.

\section{References}

Alvarado JC, Vaughan JW, Stanford TR, Stein BE (2007) Multisensory versus unisensory integration: contrasting modes in the superior colliculus. J Neurophysiol 97:3193-3205 
Amassian VE, Cracco RQ, Maccabee PJ, Cracco JB, Rudell A, Eberle L (1989) Suppression of visual perception by magnetic coil stimulation of human occipital cortex. Electroencephalogr Clin Neurophysiol 74:458-462

Andersen RA (1997) Multimodal integration for the representation of space in the posterior parietal cortex. Philos Trans R Soc Lond B Biol Sci 352:1421-1428

Beauchamp MS (2005) See me, hear me, touch me: multisensory integration in lateral occipital-temporal cortex. Curr Opin Neurobiol 15:145-153

Bolognini N, Maravita A (2007) Proprioceptive alignment of visual and somatosensory maps in the posterior parietal cortex. Curr Biol 17:1890-1895

Bolognini N, Rasi F, Coccia M, Ladavas E (2005) Visual search improvement in hemianopic patients after audio-visual stimulation. Brain 128:2830-2842

Bolognini N, Savazzi S, Bricolo E, Marzi C, Maravita A (2007) The role of superior colliculus in audio-visual integration in humans: clues from the redundant target effect. In: Cognitive Neuroscience Society Annual Meeting, New York

Bremmer F, Schlack A, Duhamel JR, Graf W, Fink GR (2001a) Space coding in primate posterior parietal cortex. Neuroimage 14:S46-S51

Bremmer F, Schlack A, Shah NJ, Zafiris O, Kubischik M, Hoffmann K, Zilles K, Fink GR (2001b) Polymodal motion processing in posterior parietal and premotor cortex: a human fMRI study strongly implies equivalencies between humans and monkeys. Neuron 29:287-296

Brozzoli C, Dematte ML, Pavani F, Frassinetti F, Farnè A (2006) Neglect and extinction: within and between sensory modalities. Restor Neurol Neurosci 24:217-232

Bushara KO, Weeks RA, Ishii K, Catalan MJ, Tian B, Rauschecker JP, Hallett M (1999) Modality-specific frontal and parietal areas for auditory and visual spatial localization in humans. Nat Neurosci 2:759-766

Calvert GA, Hansen PC, Iversen SD, Brammer MJ (2001) Detection of audio-visual integration sites in humans by application of electrophysiological criteria to the BOLD effect. Neuroimage 14:427438

Cappelletti M, Barth H, Fregni F, Spelke ES, Pascual-Leone A (2007) rTMS over the intraparietal sulcus disrupts numerosity processing. Exp Brain Res 179:631-642

Chen R, Classen J, Gerloff C, Celnik P, Wassermann EM, Hallett M, Cohen LG (1997) Depression of motor cortex excitability by low-frequency transcranial magnetic stimulation. Neurology 48:1398-1403

Clarke S, Thiran AB (2004) Auditory neglect: what and where in auditory space. Cortex 40:291-300

Downar J, Crawley AP, Mikulis DJ, Davis KD (2000) A multimodal cortical network for the detection of changes in the sensory environment. Nat Neurosci 3:277-283

Duhamel J, Colby C, Goldberg M (1991) Congruent representation of visual and somatosensory space in single neurons of monkey ventral intraparietal area (area VIP). In: Paillard J (ed) Brain and space. Oxford University Press, Oxford, pp 223-236

Duncan J, Humphreys G, Ward R (1997) Competitive brain activity in visual attention. Curr Opin Neurobiol 7:255-261

Frassinetti F, Bolognini N, Bottari D, Bonora A, Ladavas E (2005) Audiovisual integration in patients with visual deficit. J Cogn Neurosci 17:1442-1452

Gondan M, Niederhaus B, Rosler F, Roder B (2005) Multisensory processing in the redundant-target effect: a behavioral and eventrelated potential study. Percept Psychophys 67:713-726

Graziano MS, Gross CG (1998) Spatial maps for the control of movement. Curr Opin Neurobiol 8:195-201

Hyvärinen J (1981) Regional distribution of functions in parietal association area 7 of the monkey. Brain Res 206:287-303
Jiang W, Wallace MT, Jiang H, Vaughan JW, Stein BE (2001) Two cortical areas mediate multisensory integration in superior colliculus neurons. J Neurophysiol 85:506-522

Jiang W, Jiang H, Stein BE (2002) Two corticotectal areas facilitate multisensory orientation behavior. J Cogn Neurosci 14:1240 1255

Kastner S, Ungerleider LG (2000) Mechanisms of visual attention in the human cortex. Annu Rev Neurosci 23:315-341

Kastner S, Ungerleider LG (2001) The neural basis of biased competition in human visual cortex. Neuropsychologia 39:1263-1276

Knecht S, Ellger T, Breitenstein C, Bernd Ringelstein E, Henningsen $\mathrm{H}$ (2003) Changing cortical excitability with low-frequency transcranial magnetic stimulation can induce sustained disruption of tactile perception. Biol Psychiatry 53:175-179

Lewald J, Foltys H, Topper R (2002) Role of the posterior parietal cortex in spatial hearing. J Neurosci 22:RC207

Macaluso E, Driver J (2005) Multisensory spatial interactions: a window onto functional integration in the human brain. Trends Neurosci 28:264-271

Machii K, Cohen D, Ramos-Estebanez C, Pascual-Leone A (2006) Safety of rTMS to non-motor cortical areas in healthy participants and patients. Clin Neurophysiol 117:455-471

Maravita A, Savazzi S, Bricolo E, Penati V, Marzi C (2005) Role of superior colliculus in audio-visual redundancy gain. In: Sixth International Multisensory Research Forum, Rovereto Italy

Maravita A, Bolognini N, Bricolo E, Marzi CA, Savazzi S (2008) Is audiovisual integration subserved by the superior colliculus in humans? NeuroReport 19:271-275

Merabet L, Thut G, Murray B, Andrews J, Hsiao S, Pascual-Leone A (2004) Feeling by sight or seeing by touch? Neuron 42:173-179

Meredith MA, Stein BE (1986) Visual, auditory, and somatosensory convergence on cells in superior colliculus results in multisensory integration. J Neurophysiol 56:640-662

Miller J (1982) Divided attention: evidence for coactivation with redundant signals. Cog Psychol 14:247-279

Nachev P, Husain M (2006) Disorders of visual attention and the posterior parietal cortex. Cortex 42:766-773

Pascual-Leone A, Walsh V, Rothwell J (2000) Transcranial magnetic stimulation in cognitive neuroscience-virtual lesion, chronometry, and functional connectivity. Curr Opin Neurobiol 10:232237

Pavani F, Ladavas E, Driver J (2003) Auditory and multisensory aspects of visuospatial neglect. Trends Cogn Sci 7:407-414

Raab DH (1962) Statistical facilitation of simple reaction times. Trans N Y Acad Sci 24:574-590

Romei V, Murray MM, Merabet LB, Thut G (2007) Occipital transcranial magnetic stimulation has opposing effects on visual and auditory stimulus detection: implications for multisensory interactions. J Neurosci 27:11465-11472

Romero JR, Anschel D, Sparing R, Gangitano M, Pascual-Leone A (2002) Subthreshold low frequency repetitive transcranial magnetic stimulation selectively decreases facilitation in the motor cortex. Clin Neurophysiol 113:101-107

Schlack A, Sterbing-D'Angelo SJ, Hartung K, Hoffmann KP, Bremmer F (2005) Multisensory space representations in the macaque ventral intraparietal area. J Neurosci 25:4616-4625

Schröger E, Widmann A (1998) Speeded responses to audiovisual signal changes result from bimodal integration. Psychophysiology 37:755-759

Schwarz W (1994) Diffusion, superposition, and the redundant-targets effect. J Math Psychol 50:4-520

Stanford TR, Stein BE (2007) Superadditivity in multisensory integration: putting the computation in context. NeuroReport 18:787-792

Stein BE (1998) Neural mechanisms for synthesizing sensory information and producing adaptive behaviors. Exp Brain Res 123:124135 
Stein BE (2005) The development of a dialogue between cortex and midbrain to integrate multisensory information. Exp Brain Res 166:305-315

Stein BE, Meredith M (1993) The merging of the senses. MIT, Cambridge

Stein BE, Stanford TR (2008) Multisensory integration: current issues from the perspective of the single neuron. Nat Rev Neurosci 9:255-266

Stein BE, Huneycutt WS, Meredith MA (1988) Neurons and behavior: the same rules of multisensory integration apply. Brain Res 448:355-358
Talairach J, Tournoux P (1988) A co-planar stereotactic Atlas of the human brain. Thieme Verlag, Stuttgart

Vallar G, Bottini G, Paulesu E (2003) Neglect syndromes: the role of the parietal cortex. Adv Neurol 93:293-319

Wallace MT, Meredith MA, Stein BE (1992) Integration of multiple sensory modalities in cat cortex. Exp Brain Res 91:484-488

Wassermann EM (1998) Risk and safety of repetitive transcranial magnetic stimulation: report and suggested guidelines from the international workshop on the safety of repetitive transcranial magnetic stimulation, 5-7 June 1996. Electroencephalogr Clin Neurophysiol 108:1-16 